\title{
Sliding Mode Momentum Observers for Estimation of External Torques and Joint Acceleration
}

\author{
Gianluca Garofalo, Nico Mansfeld, Julius Jankowski and Christian Ott
}

\begin{abstract}
Interactions between robots and their environment give rise to external wrenches acting on the robot structure. The estimation of the resulting torques in the joints is fundamental in human-robot interaction to detect/identify collisions and perform suitable reaction strategies. Other applications may require to use the estimation for compensating the effects of the external torques within the control loop. The well-established momentum observer, which relies on proprioceptive sensors only, is usually used for these purposes. In this work, the momentum dynamics is used to derive new observers. While the classic momentum observer provides a first-order filtered version of the external torques, here a (theoretically) finite-time convergence is achieved. Simulations and experiments are used to validate the performance of the proposed methods.
\end{abstract}

\section{INTRODUCTION}

In physical human-robot interaction ( $\mathrm{pHRI}$ ), unwanted or unforeseen contact cannot be in general avoided, as the robot must act in a dynamic and partially unknown environment. Given the possibly high relative speed between the human and the robot and/or edgy surface properties of the tool or payload, accidental interactions might still result in injuries, especially in clamping situations [1]-[3].

Several precautions can be taken in order to increase the intrinsic safety of the robots. For example, the mechanical design can be centered around the reduction of the inertia and the use of compliant components [4], [5]. Similarly, control strategies can aim at realizing a compliant and safe behavior [6], [7] and avoid for possibly dangerous collisions [8], [9]. Nevertheless, all these strategies cannot always guarantee the avoidance of all contacts and therefore, it is of paramount importance to detect and identify possible interactions and handle collisions in a safe way [10]-[12].

There exist several possible solutions to detect collisions and estimate the involved external torques and wrench. A single force-torque sensor at the end-effector can measure the wrench, but is only capable of detecting collisions at the end-effector and not elsewhere along the structure. Artificial skin or airbags [13], [14] could solve this problem, but it is currently very costly or even not possible to fully equip all robots with such a solution. Monitoring the estimated external torques based on proprioceptive sensors is a popular approach that is widely used nowadays [15]. In some setups, e.g. when localizing the contact point with an exteroceptive vision system, the estimated external torques can also be used to reconstruct an estimation of the external wrench [16].

The authors are with the Institute of Robotics and Mechatronics, German Aerospace Center (DLR), Wessling, Germany. gianluca.garofalo(at)dlr.de

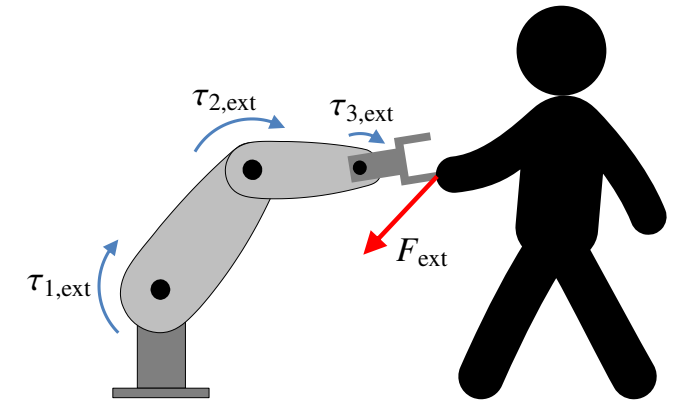

Fig. 1. When a robot is in contact with the environment, an external wrench $\boldsymbol{F}_{\text {ext }}$ is exerted on the robot structure. This wrench results in external torques $\tau_{\text {ext }}$ in the robot joints. The goal of a collision observer is to obtain a precise estimate $\hat{\tau}_{\text {ext }}$ of the real external torques.

The most well-established collision detection scheme that relies on proprioceptive torques sensors only is the so-called momentum observer, which was originally proposed in [17]. Fifteen years after its first development in the context of fault detection and identification, it has been used in different fields and with different applications [15], [18]-[20]. The success of the momentum observer goes along with the increasing diffusion of human-friendly robots. However, the momentum observer provides a first-order filtered version of the external torques and therefore achieves asymptotic convergence only for constant external torques.

In this paper, new momentum observers inspired by the one in [17] are proposed. The new observers guarantee finitetime convergence of the estimated torques to the real ones, as well as a finite-time estimation of the joint acceleration. Therefore, after a time $T$, the estimation coincides with the real signals. The finite-time behavior of the observers is obtained using sliding mode techniques [21], [22]. Furthermore, the observers can be easily extended from joint space to task space, to obtain directly an estimation of the external wrench.

The estimation of the external torques provided by the proposed observers is useful not only in pHRI scenarios, but also in other applications, like for friction compensation [18] and reactive strategies in unmanned aerial vehicles [20].

The paper is organized as follows. Section II briefly presents the dynamic model of the considered systems. In Section III, the classic first order momentum observer is recalled and afterwards the newly proposed ones are derived. In Section IV the new results are compared to the state of the art in simulation, while presenting an analysis of the parameters. Section V reports the results of the experimental comparisons. Finally, Section VI summarizes the work. 


\section{Robot Dynamics}

The systems considered in this paper are rigid-joint and rigid-link manipulators. Nevertheless, for estimating the external torques, the results obtained in the context of rigidjoint robots can be trivially extended in presence of elastic joints [15]. Therefore, the elastic case will not be considered.

The considered robotic systems are modeled by the set of nonlinear differential equations:

$$
\boldsymbol{M}(\boldsymbol{q}) \ddot{\boldsymbol{q}}+\boldsymbol{C}(\boldsymbol{q}, \dot{\boldsymbol{q}}) \dot{\boldsymbol{q}}+\boldsymbol{g}(\boldsymbol{q})=\boldsymbol{\tau}+\tau_{e},
$$

where the link positions and velocities $\boldsymbol{q}, \dot{\boldsymbol{q}} \in \mathbb{R}^{n}$ ( $n$ is the number of joints) are the state of the robot. It is used $\boldsymbol{M}(\boldsymbol{q}) \in \mathbb{R}^{n \times n}$ to denote the symmetric and positive definite inertia matrix, $\boldsymbol{C}(\boldsymbol{q}, \dot{\boldsymbol{q}}) \in \mathbb{R}^{n \times n}$ a Coriolis matrix satisfying the passivity property

$$
\dot{\boldsymbol{M}}(\boldsymbol{q}, \dot{\boldsymbol{q}})=\boldsymbol{C}(\boldsymbol{q}, \dot{\boldsymbol{q}})+\boldsymbol{C}(\boldsymbol{q}, \dot{\boldsymbol{q}})^{\top}
$$

and $\boldsymbol{g}(\boldsymbol{q}) \in \mathbb{R}^{n}$ the gravity torque vector. The torques $\tau \in \mathbb{R}^{n}$ produced by the motors are an input to the system, together with the external torques $\tau_{e}$. If external wrenches are applied to the system, then $\boldsymbol{\tau}_{e}=\boldsymbol{J}_{e}(\boldsymbol{q})^{\top} \boldsymbol{w}_{e}$, where $\boldsymbol{w}_{e} \in \mathbb{R}^{6 m}$ ( $m$ is the number of links in contact) collects the stacked wrenches and $\boldsymbol{J}_{e}(\boldsymbol{q}) \in \mathbb{R}^{n \times 6 m}$ is the corresponding Jacobian matrix.

Finally, for sake of readability, the dependencies will be dropped in the remainder of the paper.

\section{Momentum Observers}

Many schemes exist for detecting, isolating, and identifying collisions in robotic manipulators. A thorough overview and analysis of them is given in [15]. The most well established method is the so-called momentum observer [10], [17]. Despite its wide diffusion and application in scenarios even beyond human-robot interaction [18], [20], this method provides only a first-order filtered version of the disturbance. As a consequence, the user is faced with a compromise between noise attenuation and estimation performance.

The main result of this paper is inspired by the momentum observer in [10], [17], but provides a finite-time estimation of the disturbance instead of a first-order filtered version. Theoretically, the resulting estimation is therefore more accurate and it provides an exact estimation of the disturbance after a finite time $T$. The possibility of having an improved estimation is obviously beneficial also in applications that aim at compensating for the disturbance [18].

In the following, it will be first reviewed the first-order momentum observer and then presented the main contribution of this work, i.e. sliding mode momentum observers. The classic first-order observer always requires two assumptions:

1) the dynamic model is perfectly known,

2) the friction torque is negligible or known.

These assumption are necessary also for the newly designed ones. The reason is that the observers will estimate the whole torques $\tau_{e}$ in (1), i.e., all the torques which are not explained by the model. The two assumptions are, therefore, necessary to guarantee that $\tau_{e}$ is due only to the interaction with the environment and does not contain terms due to model uncertainties and additional possible disturbances. The reader is referred to [15] for detailed considerations related to this topic. In short, all these effects can be accumulated with measurement noise. Consequently, one must set an empiricallyobtained threshold to discriminate the occurrence of real collisions and decrease the probability of false positives.

The derivation of all momentum observers is based on the timely evolution of the generalized momentum of the robot

$$
\boldsymbol{p}=\boldsymbol{M} \dot{\boldsymbol{q}}
$$

Using (1), its time derivative is given by

$$
\begin{aligned}
\dot{\boldsymbol{p}} & =\boldsymbol{\tau}+\boldsymbol{\tau}_{e}-\boldsymbol{C} \dot{\boldsymbol{q}}-\boldsymbol{g}+\dot{\boldsymbol{M}} \dot{\boldsymbol{q}} \\
& =\boldsymbol{\tau}+\boldsymbol{\tau}_{e}+\boldsymbol{C}^{\top} \dot{\boldsymbol{q}}-\boldsymbol{g},
\end{aligned}
$$

where the passivity property (2) has been used.

\section{A. First-Order Momentum Observer}

The derivation of the classic first-order momentum observer is based on the residual

$$
\boldsymbol{r}=\boldsymbol{K}_{O}\left(\boldsymbol{p}-\int_{0}^{t}\left(\boldsymbol{\tau}+\boldsymbol{C}^{\top} \dot{\boldsymbol{q}}-\boldsymbol{g}+\boldsymbol{r}\right) \mathrm{d} s-\boldsymbol{p}(0)\right),
$$

where $\boldsymbol{p}(0)$ is the initial value of the generalized momentum (assuming $t_{0}=0$ as initial time) and $\boldsymbol{K}_{O}=\operatorname{diag}\left(k_{O, i}\right)>0$. The key point in the definition of $\boldsymbol{r}$ is that, by using (4), its time derivative is

$$
\dot{\boldsymbol{r}}=-\boldsymbol{K}_{O}\left(\boldsymbol{r}-\boldsymbol{\tau}_{e}\right)
$$

i.e., $n$ decoupled linear systems. This means that $\boldsymbol{r}$ is a firstorder filtered version of $\tau_{e}$ and therefore it can be used as an estimation of the external torques. Notice, nonetheless, that $\boldsymbol{r}$ will be a practically exact estimation of $\tau_{e}$ only if the latter is a constant signal and after a time given by the slowest time constant $T_{O, i}=1 / k_{O, i}$.

In practice, when selecting the observer gain, one must find a trade-off between desired monitoring bandwidth and permissible noise amplification. The first-order characteristic of the momentum observer leads to a phase lag in the estimated external torque signal (see also Section IV).

\section{B. Sliding Mode Momentum Observers}

1) SOSM Momentum Observer: As in Section III-A, the key point is to consider the generalized momentum $\boldsymbol{p}$ and the expression of $\dot{\boldsymbol{p}}$ in (4). From the latter it follows

$$
\boldsymbol{\tau}+\boldsymbol{C}^{\top} \dot{\boldsymbol{q}}-\boldsymbol{g}=\dot{\boldsymbol{p}}-\tau_{e},
$$

therefore the available left hand-side can be used in place of the unknown terms on the right hand-side to design an observer that provides an estimation of the momentum in finite-time. Furthermore, the mismatch between the estimated momentum dynamics and the real one is exactly the external torque, which consequently will be as well estimated in finite-time. The observer dynamics is allowed to have discontinuous terms. In this case, the differential equation with locally bounded Lebesgue-measurable right-hand side is understood in the sense of Filippov [23] and the absolutely continuous solutions satisfy the differential inclusion almost 
everywhere. In particular, assuming that $\boldsymbol{\tau}_{e}$ has a known global Lipschitz constant $L$, so that the signal can be not smooth, but its derivative exists almost everywhere, one can consider the observer structure

$$
\begin{aligned}
& \dot{\hat{p}}=\boldsymbol{\tau}+\boldsymbol{C}^{\top} \dot{\boldsymbol{q}}-\boldsymbol{g}-\boldsymbol{T}|\tilde{\boldsymbol{p}}|^{\frac{1}{2}} \operatorname{sgn}(\tilde{\boldsymbol{p}})+\boldsymbol{\sigma}, \\
& \dot{\boldsymbol{\sigma}}=-\boldsymbol{S} \operatorname{sgn}(\tilde{\boldsymbol{p}}),
\end{aligned}
$$

where $\tilde{\boldsymbol{p}}=\hat{\boldsymbol{p}}-\boldsymbol{p}$, all the operators in (7) have to be undersood as applied element-wise and $\boldsymbol{S}, \boldsymbol{T} \in \mathbb{R}^{n \times n}$ are positive definite diagonal matrices, such that each element on the diagonal satisfies the set of inequalities given in [22] (see also the Appendix). Using (4) and defining $s:=\sigma-\tau_{e}$, one gets the observer error dynamics

$$
\begin{aligned}
\dot{\tilde{\boldsymbol{p}}} & =-\boldsymbol{T}|\tilde{\boldsymbol{p}}|^{\frac{1}{2}} \operatorname{sgn}(\tilde{\boldsymbol{p}})+\boldsymbol{s}, \\
\dot{\boldsymbol{s}} & =-\boldsymbol{S} \operatorname{sgn}(\tilde{\boldsymbol{p}})-\dot{\boldsymbol{\tau}}_{e},
\end{aligned}
$$

i.e., $n$ indipendent super twisting algorithms (STA) [21]. Since for the STA robust, global finite-time stability of the equilibrium point $(\tilde{\boldsymbol{p}}, \boldsymbol{s})=(\mathbf{0}, \mathbf{0})$ can be guaranteed [21], [22], then $\sigma$ becomes in finite time an estimation of $\tau_{e}$.

Additionally, as $\tilde{\boldsymbol{p}}, \dot{\tilde{\boldsymbol{p}}} \rightarrow \mathbf{0}$ in finite time, it is possible to use $\dot{\hat{\boldsymbol{p}}}$ to obtain a finite-time estimation of $\ddot{\boldsymbol{q}}$, as

$$
\ddot{\hat{\boldsymbol{q}}}=\boldsymbol{M}^{-1} \dot{\hat{\boldsymbol{p}}}-\frac{\mathrm{d}}{\mathrm{d} t}\left(\boldsymbol{M}^{-1}\right) \hat{\boldsymbol{p}} .
$$

Once again, this is better than what it can be achieved by the first-order momentum observer, which does not provide an estimate of the generalized momentum.

It is worth noticing that although both methods use the momentum dynamics to obtain an estimation of $\tau_{e}$, the way in which (4) is used is quite different. In the residual $\boldsymbol{r}$, the momentum dynamics is used as integrand, while in (7) the states of the observers are directly an estimation of the generalized momentum $\boldsymbol{p}$ and external torque $\tau_{e}$.

In the nominal case, the observer guarantees finite-time convergence to $\tau_{e}$, but in real case scenarios model uncertainties, measurement noise, sampling time, and chattering effect will inevitably lead to a noisy estimation. As a consequence, just like in Section III-A, the estimated signal will not be exactly zero even when no contact is present and a threshold has to be empirically set to avoid false positives.

2) SOSML Momentum Observer: Having understood the basic idea leading to the SOSM momentum observer, the result can be further improved by introducing linear terms in (7). In this way, one obtains the observer

$$
\begin{aligned}
& \dot{\hat{\boldsymbol{p}}}=\boldsymbol{\tau}+\boldsymbol{C}^{\top} \dot{\boldsymbol{q}}-\boldsymbol{g}-\boldsymbol{T}_{1}|\tilde{\boldsymbol{p}}|^{\frac{1}{2}} \operatorname{sgn}(\tilde{\boldsymbol{p}})-\boldsymbol{T}_{2} \tilde{\boldsymbol{p}}+\boldsymbol{\sigma}, \\
& \dot{\boldsymbol{\sigma}}=-\boldsymbol{S}_{1} \operatorname{sgn}(\tilde{\boldsymbol{p}})-\boldsymbol{S}_{2} \tilde{\boldsymbol{p}} .
\end{aligned}
$$

The error dynamics of this observer is $n$ independent second order sliding mode (SOSML) introduced in [22], for which robust, global finite-time stability of $(\tilde{\boldsymbol{p}}, \boldsymbol{s})=(\mathbf{0 , 0})$ is guaranteed if the elements on the diagonals of the positive definite diagonal matrices $\boldsymbol{S}_{i}, \boldsymbol{T}_{i}$ satisfy the inequalities given in [22] (see also the Appendix). The advantage of the SOSML over the SOSM is in the behavior of the system far from the equilibrium point in the origin. While the effect of the nonlinear terms is stronger than the linear ones near the equilibrium point (resulting in a finite-time rather than exponential convergence), the linear terms are predominant the farther the initial condition is from the origin.

\section{Parameter Analysis and Simulation Results}

\section{A. First-Order Momentum Observer}

When selecting the observer gain, one must find a tradeoff between desired monitoring bandwidth and noise amplification. In order to achieve $\boldsymbol{r} \approx \boldsymbol{\tau}_{e}, \boldsymbol{K}_{O}$ should be as large as possible. However, large values for $\boldsymbol{K}_{O}$ also result in higher noise amplification in the estimated torque, which deteriorates the observer performance in terms of sensitivity.

To gain an intuition on the role of $\boldsymbol{K}_{O}$ in the noise amplification, assume that:

- the main uncertainty is due to noise in the measured joint velocity $\dot{\boldsymbol{q}}_{m e s}=\dot{\boldsymbol{q}}+\boldsymbol{\epsilon}$, and

- the noise $\boldsymbol{\epsilon}$ in the measured joint velocity has a high frequency and is zero-mean, such that the effect on the integral $\hat{\boldsymbol{p}}:=\int_{0}^{t}\left(\boldsymbol{\tau}+\boldsymbol{C}^{\top} \dot{\boldsymbol{q}}_{m e s}-\boldsymbol{g}+\boldsymbol{r}\right) \mathrm{d} s$ is negligible.

In these conditions, the difference between the ideal residual $\boldsymbol{r}$ and $\boldsymbol{r}_{o b s}$ obtained in presence of noise is:

$$
\Delta \boldsymbol{r}_{\epsilon}=\boldsymbol{r}_{o b s}-\boldsymbol{r}=\boldsymbol{K}_{O}\left(\boldsymbol{M} \dot{\boldsymbol{q}}_{m e s}-\boldsymbol{M} \dot{\boldsymbol{q}}\right)=\boldsymbol{K}_{O} \boldsymbol{M} \boldsymbol{\epsilon} .
$$

The error due to a noisy velocity is thus amplified by both the observer gain $\boldsymbol{K}_{O}$ and the inertia matrix $\boldsymbol{M}$. For illustrating the influence of $\boldsymbol{K}_{O}$ on the dynamic performance and noise behavior, a 1-DOF joint was simulated. Typical values for $K_{O}$ were used, namely 20, 50, and 80 in Hz. In Fig. 2 (left), we analyze the influence of the gain and the link mass $(1 \mathrm{~kg}$ and $4 \mathrm{~kg}$ ) on noise amplification. The noise of the velocity signal is zero mean Gaussian noise with $50 \mathrm{nW}$ power at $1 \mathrm{kHz}$ sampling rate, which is comparable to the one in the DLR/KUKA LWR III/IV. In agreement with (11), the noise amplitude is proportional to both $K_{O}$ and the link inertia. The influence of $K_{O}$ on phase lag and amplitude (in absence of noise) is depicted in Fig. 3 (left). Therefore, the larger bandwidth obtained by increasing $K_{O}$, comes at the price of a higher threshold for avoiding the occurrence of false positives in the collision detection due to the noise amplification. Finally, for a given value of the observer gain and increasing noise power, Fig. 4 (left) shows that the phase lag remains unaffected and only the noise level increases.

\section{B. SOSM and SOSML Momentum Observers}

For a successful implementation and application of the proposed sliding mode momentum observers, it is important to know how the gains $\boldsymbol{T}, \boldsymbol{S}$, and $\boldsymbol{T}_{i}, \boldsymbol{S}_{i}$, respectively, affect not only the stability, but also the dynamic behavior. The stability is ensured by satisfying the inequalities given in [22] (see also the Appendix). For selecting the gains of the SOSM momentum observer one may first choose the parameter $S$ and then the parameter $\boldsymbol{T}$. Since $\boldsymbol{S}$ is the absolute value of the slope of $\sigma$ (see (7)), it determines the tracking capability. If the slope of the external torque is higher than $\sigma$, then the observer is not capable of tracking the reference signal 
First Order (FO)

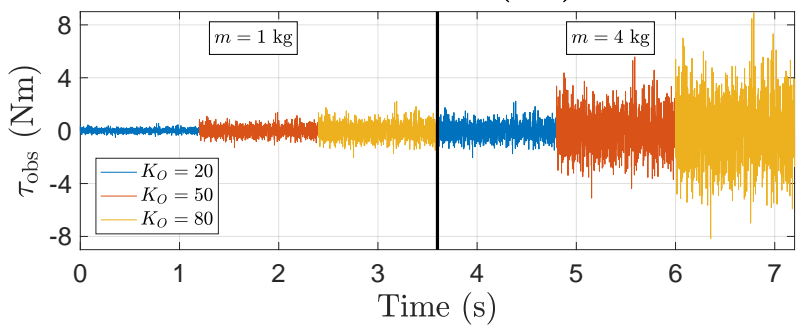

Sliding Mode (SOSML)

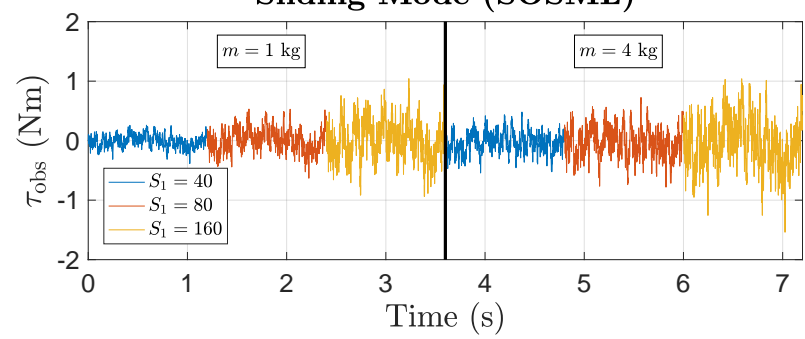

Fig. 2. Influence of observer parameters $K_{O}$ and $S_{1}$ on noise amplification. The case of a single joint for mass values of $m=1 \mathrm{~kg}$ and $4 \mathrm{~kg}$ was considered. The remaining parameters of the SOSML observer are: $T_{1}=2 \sqrt{S_{1}}, S_{2}=1600 \mathrm{~Hz}^{2}, T_{2}=80 \mathrm{~Hz}$.
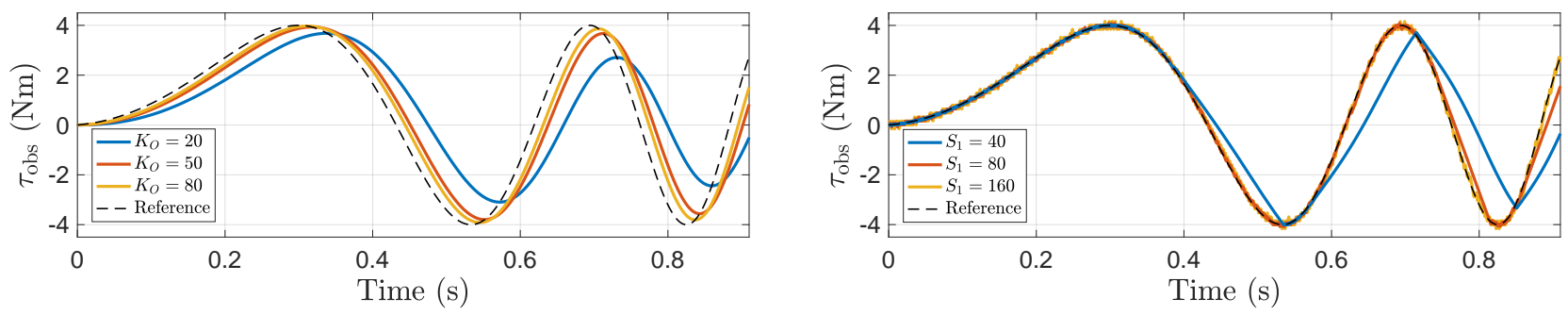

Fig. 3. Influence of observer parameters $K_{O}$ and $S_{1}$ on the tracking performance for a single joint with mass $m=1 \mathrm{~kg}$ without measurement noise. The remaining parameters of the SOSML observer are: $T_{1}=2 \sqrt{S_{1}}, S_{2}=1600 \mathrm{~Hz}^{2}, T_{2}=80 \mathrm{~Hz}$.
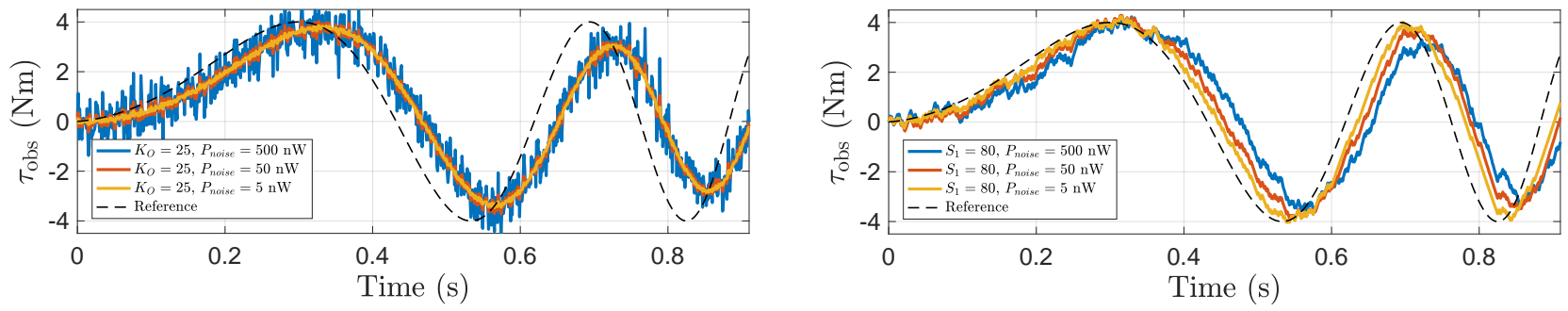

Fig. 4. Influence of measurement noise power $P_{\text {noise }}$ on the tracking performance and on the estimation noise for a single joint with mass $m=1 \mathrm{~kg}$. The remaining parameters of the SOSML observer are: $T_{1}=2 \sqrt{S_{1}}, S_{2}=1600 \mathrm{~Hz}^{2}, T_{2}=80 \mathrm{~Hz}$.

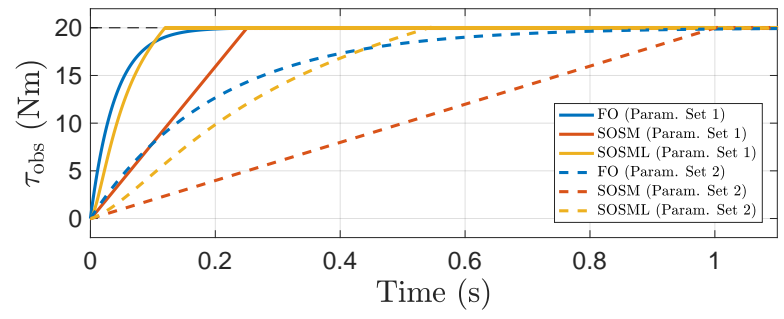

Fig. 5. Comparison of the step response of the first order observer (FO) and the proposed sliding mode observers (SOSM and SOSML). All simulations have been carried out with a single joint with mass $m=1 \mathrm{~kg}$. Parameter set 1: $K_{0}=25 \mathrm{~Hz}(\mathrm{FO}) ; S=80 \mathrm{~Hz}^{2}, T=22.4 \mathrm{~Hz}(\mathrm{SOSM})$; $S_{1}=80 \mathrm{~Hz}^{2}, T_{1}=17.9 \mathrm{~Hz}, S_{2}=1600 \mathrm{~Hz}^{2}, T_{2}=80 \mathrm{~Hz}$ (SOSML).

Parameter set $2: K_{0}=5 \mathrm{~Hz}(\mathrm{FO}) ; S=20 \mathrm{~Hz}^{2}, T=11.2 \mathrm{~Hz}(\mathrm{SOSM})$; $S_{1}=20 \mathrm{~Hz}^{2}, T_{1}=8.9 \mathrm{~Hz}, S_{2}=64 \mathrm{~Hz}^{2}, T_{2}=16 \mathrm{~Hz}(\mathrm{SOSML})$.

anymore. This is reflected by the requirement for $\tau_{e}$ to have a known global Lipschitz constant $L$, which is used in the selection of the gain $S$.

In order to obtain an intuition on how to tune the parameter $\boldsymbol{T}$, the error dynamics in (8) can be rewritten as the second order differential equation

$$
\dot{\tilde{\boldsymbol{p}}}=-\boldsymbol{T}|\tilde{\boldsymbol{p}}|^{\frac{1}{2}} \operatorname{sgn}(\tilde{\boldsymbol{p}})-\boldsymbol{S} \int_{0}^{t} \operatorname{sgn}(\tilde{\boldsymbol{p}}) \mathrm{d} s-\tau_{e},
$$

which can be interpreted as a non-linear PI controller. The parameter $\boldsymbol{T}$ can now be interpreted as the equivalent of a proportional gain, while the parameter $\boldsymbol{S}$ as the equivalent of the integral gain. Too high values for $\boldsymbol{T}$ will lead to overshoot, while too small values result in poor convergence. Empirically, starting with a value of $\boldsymbol{T}=1.6 \sqrt{\boldsymbol{S}}$ and tuning the gain is a reasonable way to obtain a desired behavior.

For the SOSML momentum observer, the linear terms correspond to those of a as second order linear filter. Thus, $\boldsymbol{T}_{2}$ and $\boldsymbol{S}_{2}$ can be chosen, for example, such that critical damping is achieved. The parameter $S_{1}$ can be designed analogously to $S$ in the SOSM momentum observer since the correction action of the linear terms is negligible in the vicinity of the equilibrium point. Again, the parameter $\boldsymbol{T}_{1}$ can be initialized with $\boldsymbol{T}_{1}=1.6 \sqrt{\boldsymbol{S}_{1}}$ and then tuned.

To compare the SOSM and SOSML momentum observers, it is considered their step response, as well as the step re- 
sponse of the first-order momentum observer. The parameters $\boldsymbol{S}_{2}, \boldsymbol{T}_{2}$ of the SOSML momentum observer are selected such that the equivalent second-order linear system has the same cutting frequency as the first-order momentum observer. Fig. 5 shows that, although the convergence of the SOSM momentum observer happens in finite time, the estimate grows linearly towards the real value. In contrast, the classic first-order momentum observer converges exponentially with a time constant independent of the amplitude of the real signal. By using the SOSML approach, the drawback of the SOSM momentum observer is overcome as the observer behaves similar to a linear filter far from the equilibrium. In this way, the estimation grows exponentially towards the real value and with the robustness and accuracy guaranteed by a finite-time convergence.

Because the SOSML momentum observer generally outperforms the SOSM momentum observer, we will only consider the former in the remainder of this paper.

\section{Properties of the SOSML Momentum Observer and Com- parison to the First-Order Momentum Observer}

Like for the first-order momentum observer, the following points will be analyzed also for the SOSML version:

- influence of observer gains and link mass on noise amplification, see Fig. 2 (right),

- convergence to reference signal in absence of noise, see Fig. 3 (right),

- influence of varying velocity noise on tracking performance, see Fig. 4 (right).

Due to all the different sources of uncertainty and the switching nature of the observer related to the limited sampling rate $^{1}$, the estimation signal is a triangular wave centered at the real signal. The amplitude of the triangular wave depends on the sampling time and the parameter $S_{1}$. On the other hand, the influence of $S_{1}$ on the noise amplification ${ }^{2}$ is illustrated in Fig. 2 (right). The noise amplitude is (slightly) proportional to $S_{1}$, but in contrast to the first-order filter, the observer seems to be almost insensitive to the value of the link mass.

In Fig. 3 (right), it is shown the ability of the SOSML momentum observer to track a reference signal (in absence of noise) for varying values of $S_{1}$. Here, the linear terms were parameterized to have the same time constant as the firstorder momentum observer with gain $K_{O}=25 \mathrm{~Hz}$. As long as the slope of the external torque is below the one $S_{1}$ was designed for, then the estimation converges to the reference in finite time. Therefore, the tracking performance improves with increasing $S_{1}$. However, the estimation becomes noisier due to the switching behavior of the scheme.

Finally, Fig. 4 (right) shows the deterioration of the tracking performance of the SOSML momentum observer, for a constant value of the observer parameters and varying power of the velocity noise. In the considered example,

\footnotetext{
${ }^{1}$ Simulatons are implemented using a simple Euler method.

${ }^{2} T_{1}$ was selected such that no overshoot occurs. The linear terms of the SOSML have negligible effect on the noise level.
}

the phase lag increases and the amplitude decreases for increasing noise power. However, in contrast to the firstorder momentum observer, the noise on the estimation signal remains almost unaltered.

The comparison between the first-order momentum observer and the SOSML momentum observer can be summarized with the following qualitative observations:

- the estimation noise for the state-of-the-art technique is proportional to $K_{O}$ and the inertia of the system. On the contrary, the noise level in the SOSML momentum observer depends only on the selected gains $\left(S_{1}\right)$,

- the dynamic response of the first-order momentum observer is mainly affected by its gain and not by a noisy velocity. For the SOSML momentum observer, both have an influence,

- the amplitude of the estimation noise is proportional to the velocity noise power for the classic momentum observer, but not for the SOSML one.

\section{ExPERIMENTAL VALIDATION}

To further compare the SOSML momentum observer and the classic first-order momentum observer, two experiments were carried out. The setup consists of a DLR/KUKA Lightweight Robot IV (LWR) and a ME-Systeme KD24s 1-DOF force sensor attached to the end-effector of the robot. The sensor was used as ground truth. All signals were recorded at $1 \mathrm{kHz}$ sampling rate. For the first-order momentum observer, the gain was selected as $K_{0}=25 \mathrm{~Hz}$ for each joint. The SOSML momentum observer parameters were $S_{1}=80 \mathrm{~Hz}^{2}, T_{1}=17.9 \mathrm{~Hz}, S_{2}=1600 \mathrm{~Hz}^{2}$, and $T_{2}=80 \mathrm{~Hz}^{2}$. To make the comparison of both observers as fair as possible, the linear terms $S_{2}, T_{2}$ were selected such that both observers have a similar dynamic response. Then, the non-linear terms $S_{1}, T_{1}$ were tuned to match the noise level in the estimation signals of the two methods. In this way, the first-order momentum observer could be replaced by the SOSML one, while keeping the same collision thresholds.

In the first experiment, it was evaluated the capability of the observers to estimate a sinusoidal external force at the end-effector. The robot was put in a configuration where the end-effector is pointing downwards. A mass-spring system was attached to the sensor and given an initial displacement to induce the sinusoidal motion. The recorded sensor and estimation signals are shown in Fig. 6. The result indicates that the estimation of both observers is very similar, with the SOSML momentum observer being slightly faster than the first-order momentum observer in this experiment.

The second experiment shows the detection of a collision. The robot was hit at the force sensor by a padded impactor. Fig. 7 shows (as in [15]) the timing properties of the force signal, the observer residuals, and the collision detection signals. In terms of the time required to detect the collision (with threshold set at $3 \mathrm{Nm}$ ), both observers have similar performance. Here, the first-order momentum observer is one time step faster than the SOSML momentum observer. 

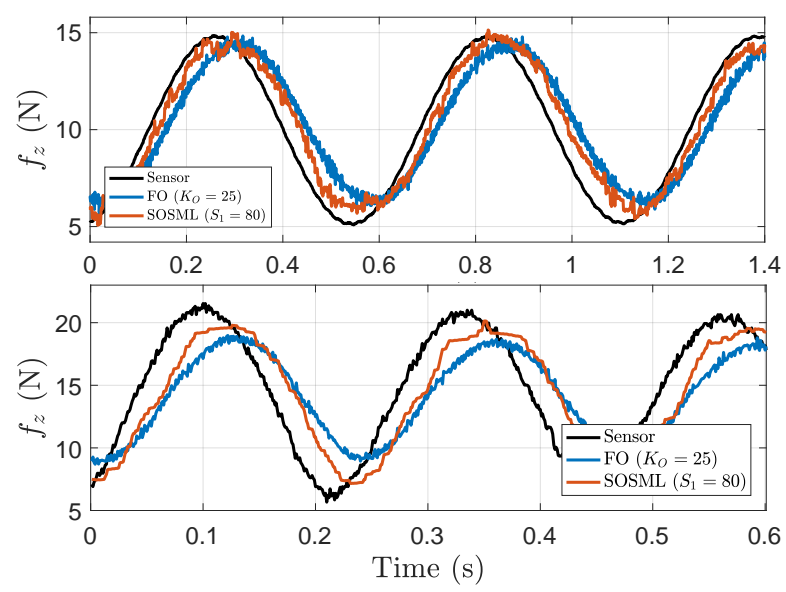

Fig. 6. Estimated Cartesian force of the first-order (blue) and SOSML (red) observers, with force sensor signal (black) for two mass-spring experiments. The excitation frequencies are approximately $1.8 \mathrm{~Hz}$ and $4.4 \mathrm{~Hz}$.

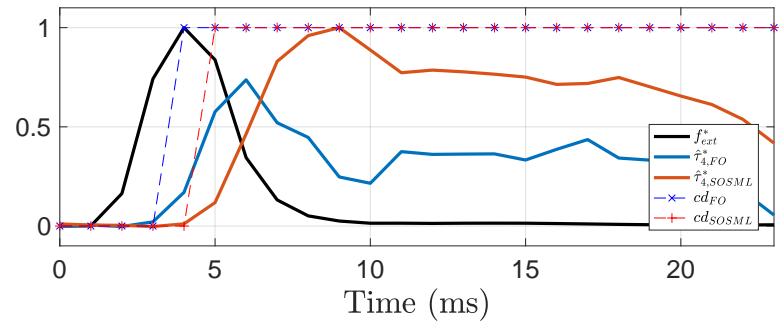

Fig. 7. Timing properties of force estimation and collision detection. The solid blue and red line represent the torque estimations $\hat{\tau}_{4, F O}^{*}$ and $\hat{\tau}_{4, S O S M L}^{*}$ of the fourth joint. Both signals were normalized w.r.t. the maximum of $\hat{\tau}_{4, S O S M L}$. The black line is the external force $f_{e x t}^{*}$, normalized w.r.t. its maximum. The dashed red and blue lines are the collision detection signals.

\section{Conclusion}

The so-called momentum observer is widely used in the robotics community due to the many scenarios in which it is required an estimation of the external wrenches arising during interactions with the environment. Nevertheless, the classic momentum observer provides an exact estimation only when the external torques are constant. This is a consequence of the observer structure, which leads to $n$ independent first-order filters for the estimation. In this work, two observers were proposed, which are capable (theoretically) of estimating in finite-time any external torques as long as the signals have a known global Lipschitz constant. The class of disturbances for which the new observers guarantee good performance is therefore greatly larger. This is possible by relying on sliding mode techniques for the design of the observer dynamics. Most importantly, the improvement comes with no additional prerequisite on the robot and signal properties compared to the state-of-the-art approach.

The role of the parameters of both classic and newly proposed observers was explained. In particular, it was analyzed their influence on (velocity) noise amplification and signal tracking performance. Experiments with a DLR/KUKA Lightweight Robot IV and simulations verified the effectiveness of the sliding mode momentum observer, which achieved similar or even slightly better results than the classic scheme. As the results depend on many factors, such as measurement noise, modeling errors, robot mass distribution, observer parameters and so on, at this point it was not possible to draw a definitive conclusion on which observer is superior for a certain class of systems. On the one hand, the superiority of the proposed method is thoroughly understood and verified in the nominal case without model uncertainties and measurement noise. On the other hand, the influence of the latter on the estimated torques requires further analysis to be addressed in future works.

\section{APPENDIX}

The following is taken from [22] and the case of an STA can be easily obtained by setting the linear gains to zero.

Theorem 1 ( [22]): Given the differential inclusion

$$
\begin{aligned}
& \dot{x}_{1}=-k_{1}\left|x_{1}\right|^{\frac{1}{2}} \operatorname{sgn}\left(x_{1}\right)-k_{2} x_{1}+x_{2}+\rho_{1}(t, x) \\
& \dot{x}_{2}=-k_{3} \operatorname{sgn}\left(x_{1}\right)-k_{4} x_{1}+\rho_{2}(t, x),
\end{aligned}
$$

suppose that the perturbation terms are globally bounded by

$$
\left|\rho_{1}\right| \leq \delta_{1}\left|x_{1}\right|^{\frac{1}{2}}+\delta_{3}\left|x_{1}\right|, \quad\left|\rho_{2}\right| \leq \delta_{2}+\delta_{4}\left|x_{1}\right|,
$$

for some known constants $\delta_{i} \geq 0, i \in\{1,2,3,4\}$. If the gains satisfy the following set of inequalities for which there always exists a solution for every $\delta_{i}$

$$
\begin{aligned}
& k_{1}>2 \max \left(\delta_{1}, \sqrt{\delta_{2}}\right), \quad k_{2}>\frac{3}{8} \delta_{3}+\frac{1}{4} \sqrt{\frac{9}{4} \delta_{3}^{2}+8 \delta_{4}} \\
& k_{3}>k_{1} \frac{\delta_{1} k_{1}+\frac{1}{8} \delta_{1}^{2}+\delta_{2}}{2\left(\frac{1}{2} k_{1}-\delta_{1}\right)} \\
& k_{4}>\frac{k_{1}\left[p_{2}\left(2 k_{2}^{2}-\frac{3}{2} \delta_{2} k_{2}-\delta_{4}\right)+\left(\frac{5}{2} k_{2}^{2}+\frac{3}{2} \delta_{3} k_{2}+\delta_{4}\right) p_{1}\right]}{2\left(p_{1}-p_{2}\right)\left(\frac{1}{2} k_{1}-\delta_{1}\right)}-\frac{1}{2} k_{2}^{2}
\end{aligned}
$$

and

$$
\begin{aligned}
& k_{2}>2 \delta_{3}, \quad k_{4}>k_{2} \frac{k_{2}\left(k_{2}+3 \delta_{3}\right)+\frac{1}{2} \delta_{3}^{2}+\delta_{4}}{p_{3}} \\
& k_{3}>\frac{\left(k_{2} \delta_{1}+\frac{1}{2} k_{1} \delta_{3}\right)^{2}}{2 k_{2} p_{3}}+\frac{\delta_{2} k_{2}+\frac{3}{2} \delta_{1} k 1 k_{2}-2 k_{1}^{2} k_{2}+\frac{1}{2} \delta_{3} k_{1}^{2}}{p_{3}}
\end{aligned}
$$

where

$$
\begin{aligned}
& p_{1}=k_{1}\left(\frac{1}{4} k_{1}^{2}-\delta_{2}\right)+\left(\frac{1}{4} k_{1}-\delta_{1}\right)\left(2 k_{3}+\frac{1}{2} k_{1}^{2}\right) \\
& p_{2}=\frac{1}{2} k_{1}\left(k_{1}+\frac{1}{2} \delta_{1}\right)^{2}, \quad p_{3}=k_{2}-2 \delta_{3}
\end{aligned}
$$

then the origin is an equilibrium point that is strongly globally asymptotically stable and all trajectories converge to it in finite time.

\section{ACKNOWLEDGMENT}

This work was partially supported by the European Commission (H2020-ICT-645097 COMANOID). 


\section{REFERENCES}

[1] S. Haddadin, A. Albu-Schäffer, and G. Hirzinger, "Requirements for safe robots: Measurements, analysis \& new insights," Int. Journal of Robotics Research, vol. 28, no. 11-12, pp. 1507-1527, 2009.

[2] S. Haddadin, A. Albu-Schäffer, and G. Hirzinger, "Soft-tissue injury caused by sharp tools: Definitions, experiments and countermeasures," IEEE Robotics and Automation Magazine, pp. 20-34, 2011.

[3] N. Mansfeld, M. Hamad, M. Becker, A. G. Marin, and S. Haddadin, "Safety map: A unified representation for biomechanics impact data and robot instantaneous dynamic properties," IEEE Robotics and Automation Letters, vol. 3, no. 3, pp. 1880-1887, 2018.

[4] A. Bicchi and G. Tonietti, "Fast and soft arm tactics: Dealing with the safety-performance trade-off in robot arms design and control," IEEE Robotics and Automation Magazine, vol. 11, pp. 22-33, 2004.

[5] J.-J. Park, B.-S. Kim, J.-B. Song, and H.-S. Kim, "Safe link mechanism based on nonlinear stiffness for collision safety," Mechanism and Machine Theory, vol. 43, no. 10, pp. 1332-1348, 2008.

[6] S. Haddadin, S. Haddadin, A. Khoury, T. Rokahr, S. Parusel, R. Burgkart, et al., "On making robots understand safety: Embedding injury knowledge into control," Int. Journal of Robotics Research, vol. 31, no. 13, pp. 1578-1602, 2012.

[7] B. Lacevic, P. Rocco, and A. M. Zanchettin, "Safety assessment and control of robotic manipulators using danger field," vol. 29, no. 5, pp. 1257-1270, 2013.

[8] O. Khatib, "Real-time obstacle avoidance for manipulators and mobile robots," in Autonomous robot vehicles. Springer, 1986, pp. 396-404.

[9] F. Flacco, T. Kröger, A. De Luca, and O. Khatib, "A depth space approach to human-robot collision avoidance," in Robotics and Automation (ICRA), 2012 IEEE International Conference on. IEEE, 2012, pp. 338-345.

[10] A. de Luca and R. Mattone, "Sensorless robot collision detection and hybrid force/motion control," in IEEE Int. Conf. on Robotics and Automation (ICRA), Barcelona, Spain, Apr. 2005, pp. 999-1004.

[11] A. De Luca, A. Albu-Schäffer, S. Haddadin, and G. Hirzinger, "Collision detection and safe reaction with the DLR-III lightweight

[22] J. A. Moreno and M. Osorio, "A Lyapunov approach to second-order sliding mode controllers and observers," in IEEE Conf. on Decision and Control, Cancun, Mexico, Dec. 2008, pp. 2856-2861. manipulator arm," in IEEE/RSJ Int. Conf. on Intelligent Robots and Systems (IROS), 2006, pp. 1623-1630.

[12] S. Haddadin, A. Albu-Schäffer, A. D. Luca, and G. Hirzinger, "Collision detection \& reaction: A contribution to safe physical human-robot interaction," in IEEE/RSJ Int. Conf. on Intelligent Robots and Systems (IROS), 2008, pp. 3356-3363.

[13] M. I. Tiwana, S. J. Redmond, and N. H. Lovell, "A review of tactile sensing technologies with applications in biomedical engineering," Sensors and Actuators A: Physical, vol. 179, pp. 17-31, 2012.

[14] R. Weitschat, J. Vogel, S. Lantermann, and H. Höppner, "End-effector airbags to accelerate human-robot collaboration," in IEEE Int. Conf. on Robotics and Automation (ICRA), Singapore, Singapore, May 2017 , pp. 2279-2284

[15] S. Haddadin, A. De Luca, and A. Albu-Schäffer, "Robot collisions: A survey on detection, isolation, and identification," IEEE Trans. on Robotics, vol. 33, no. 6, pp. 1292-1312, 2017.

[16] E. Magrini, F. Flacco, and A. De Luca, "Estimation of contact forces using a virtual force sensor," in IEEE/RSJ Int. Conf. on Intelligent Robots and Systems (IROS). IEEE, 2014, pp. 2126-2133.

[17] A. de Luca and R. Mattone, "Actuator failure detection and isolation using generalized momenta," in IEEE Int. Conf. on Robotics and Automation (ICRA), Taipei, Taiwan, Sept. 2003, pp. 634-639.

[18] L. Le Tien, A. Albu-Schäffer, A. De Luca, and G. Hirzinger, "Friction observer and compensation for control of robots with joint torque measurement," in IEEE/RSJ Int. Conf. on Intelligent Robots and Systems (IROS), Nice, France, Sept. 2008, pp. 3789-3795.

[19] E. Magrini, F. Flacco, and A. De Luca, "Control of generalized contact motion and force in physical human-robot interaction," in IEEE Int. Conf. on Robotics and Automation (ICRA), 2015, pp. 2298-2304.

[20] T. Tomić, C. Ott, and S. Haddadin, "External wrench estimation, collision detection, and reflex reaction for flying robots," IEEE Trans. on Robotics, vol. 33, no. 6, pp. 1467-1482, 2017.

[21] A. Levant, "Sliding order and sliding accuracy in sliding mode control," International Journal of Control, vol. 58, no. 6, pp. 1247$1263,1993$.

[23] A. F. Filippov, Differential equations with discontinuous righthand sides, ser. Mathematics and its applications. Dordrecht: Kluwer Academic, 1988 